\title{
Termos utilizados por cantores para identificar distorções vocais intencionais
}

\author{
MAURO BARRO FIUZA* \\ MARTA ASSUMPÇÃO DE ANDRADA E SILVA**
}

\begin{abstract}
RESUMO: Distorções vocais intencionais são efeitos sonoros que se assemelham a sons ásperos, roucos ou mesmo rosnados e são utilizadas por cantores de diversos gêneros musicais como formas de expressividade ou por questões estéticas. Cantores diferentes produzem e sentem os sons de formas diferentes e, consequentemente, nomeiam esses ajustes da maneira que acreditam ser as mais adequadas. Esta pesquisa verificou como cantores, professores de canto e pesquisadores nomeiam os ajustes de distorção vocal intencional. Foi aplicado questionário online em cantores sobre os termos adotados para denominar os diferentes tipos de distorçôes vocais. Como resultado, 121 cantores responderam a enquete e mencionaram 78 nomes para os sons distorcidos que produzem. A falta de consenso sobre como esses ajustes vocais são chamados dificulta a comunicação entre músicos e demais profissionais da voz. Os termos mais vezes mencionados foram: drive, growl, gutural, creaky, voz rasgada e scream.
\end{abstract}

PALAVRAS-CHAVE: Voz cantada; Distorção vocal; Drive; Growl; Técnica vocal.

\section{Terms used by singers to identify intentional vocal distortions in Brazil}

\begin{abstract}
Intentional vocal distortions are effects that resemble harsh, hoarse or even growling sounds and are used by singers of several musical genres as forms of expressiveness or for aesthetic reasons. Different singers produce and feel these sounds in different ways and for that reason they name these voices as they believe to be the most appropriate. This research investigated how the singers, teachers of singing and researchers in Brazil name the intentional vocal distortions. An online survey was applied to singers about the terms adopted to name the different types of vocal distortions. As a result, 121 singers responded and mentioned 78 names for the distorted sounds they produce. The lack of consensus on how
\end{abstract}

\footnotetext{
* Mauro Barro Fiuza é cantor e professor de canto popular. Mestre em fonoaudiologia pela PUC-SP, com pesquisa na área de Voz. Certificado no curso The Science of the Singing Voice do Professor Johan Sundberg na Suécia. Possui aprimoramento - Formação Integrada em Voz pelo Centro de Estudos da Voz - CEV (2013), graduação em Licenciatura em Música pelo Centro Universitário SantAnna (2012). Integrante do Grupo de Estudos Vocal SP. Ministra cursos e workshops sobre técnica e ciência vocal e escreve sobre canto e ciência vocal em site próprio (www.estudiodevoz.com.br). E-mail: mauro.bf@gmail.com.

* Marta Assumpção de Andrada e Silva é fonoaudiólóga clínica, especialista em voz e em motricidade orofacial e coach em competência comunicativa e midia traning. Mestre em Distúrbios da Comunicação e Doutora em Comunicação e Semiótica ambos pela Pontifícia Universidade Católica de São Paulo (PUC-SP). Coordenadora do Programa de Estudos Pós-graduados em Fonoaudiologia da PUC-SP, professora adjunto no Curso de Fonoaudiologia da Faculdade de Ciências Médicas da Santa Casa de São Paulo e coordenadora, junto com o Prof. Dr. André Duprat, do Ambulatório de Artes Vocais da Santa Casa de São Paulo. E-mails: m.andradaesilva@gmail.com; martaandrada@pucsp.br.
} 
these vocal adjustments are called makes communication between musicians and other voice professionals difficult. The most frequently mentioned terms were drive, growl, guttural, creaky, ripped voice and scream.

KEYWORDS: Singing voice; Vocal distortion; Drive; Growl; Vocal technique.

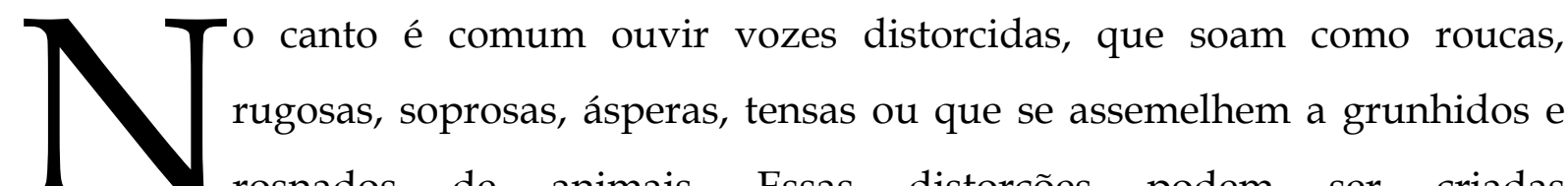
rosnados de animais. Essas distorções podem ser criadas intencionalmente por cantores habilidosos e não são necessariamente resultado de má técnica, esforço ou presença de alteração vocal como muitos podem pensar.

Esses tipos de ajuste de voz podem estar presentes em diferentes gêneros musicais, como o rock, metal, gospel, pop, sertanejo, samba, (GUZMAN ET AL., 2014), música tradicional de diversas regiões no planeta (GRAWUNDER, 2010; YOSHINAGA E KONG, 2012), aparecendo também no rap (POMPEU E BARREIRO, 2016) e no canto erudito e experimental (NEUBAUER ET AL.2004).

Entretanto, ao ouvir os cantores que utilizam esses efeitos fica claro que eles não soam todos da mesma forma, mas possuem timbres característicos distintos uns dos outros; até mesmo em um único gênero, cantores podem produzir essas distorções vocais de formas distintas. Investigações sobre como a voz é produzida indicam que existem diferentes estruturas que podem ser acionadas no aparelho fonador para produzir vozes distorcidas, e ao acionar cada uma dessas estruturas o cantor cria resultados sonoros distintos (MCGLASHAN ET AL. 2013).

Atualmente existem métodos de ensino de canto que desenvolvem práticas para o treinamento de vocais distorcidos de forma intencional, e cada um desses métodos cria sua própria terminologia (CROSS, 2007; VENDERA, 2013; SADOLIN, 2012; COELHO, 2017). Além disso, cantores e professores de canto em escolas ou estúdios particulares utilizam palavras ou expressões que acreditam serem as mais adequadas para cada sonoridade que conseguem produzir ou ensinar. Essa diversidade cria um cenário que dificulta a comunicação entre cantores de escolas diferentes e mesmo entre pesquisadores, que podem optar por utilizar um mesmo termo para diferentes ajustes vocais de acordo com sua fonte de informações. 
Em publicações de periódicos científicos é possível encontrar termos como: intentional distortions (TITZE, 1998), dist'tones (BORCH ET AL., 2004), growl (SAKAKIBARA ET AL., 2007; GUZMAN ET AL., 2014), scream (IZDEBSKI, 2017), entre outros. Neste estudo foi escolhida a expressão distorções vocais intencionais (DVIs), baseado em Titze (1998), como termo guarda-chuva para abranger todas as formas de efeitos vocais do tipo.

Para compreender o mundo das vozes distorcidas é importante conhecer como essas são denominadas pelos cantores que as utilizam. Assim será possível avaliar a complexidade do tema e entender até que ponto os estudos feitos na área respondem às necessidades dos profissionais da voz.

\section{Objetivo e método}

O objetivo deste estudo foi verificar como cantores, professores de canto e pesquisadores nomeiam os ajustes de distorção vocal intencional (DVI).

Um questionário foi elaborado para ser respondido na plataforma Google Formulários e ficou disponível para respostas dos participantes no período de maio a junho de 2017. Esse questionário foi enviado para cantores de todo o Brasil através de grupos e páginas de canto e técnica vocal em redes sociais, além de contatos pessoais do pesquisador, que é professor de canto especializado no assunto. Também foi solicitado aos participantes da pesquisa que repassassem a enquete a cantores conhecidos. O critério de inclusão foi que esses fossem cantores utilizassem qualquer tipo de distorção vocal intencional em suas atividades musicais.

Os participantes tiveram que se identificar e responder as seguintes questões: iniciais do nome, data de nascimento, cidade/estado, sexo, três gêneros musicais que canta. Foi solicitado que nomeassem com as palavras que estavam acostumados todos os tipos de distorções vocais intencionais (DVIs) que utilizam no canto.

As respostas foram tabuladas e analisadas de forma descritiva. 


\section{Resultados}

Foram recebidas 121 respostas, de cantores de 12 estados brasileiros, além de uma resposta do Chile, como visto na Tabela 1.

Tabela 1 - Distribuição dos participantes segundo estado em que reside

\begin{tabular}{lcc}
\hline Estado & Número de participantes & $\%(\mathrm{n}=121)$ \\
\hline São Paulo & 75 & $62,0 \%$ \\
Minas Gerais & 16 & $13,2 \%$ \\
Rio Grande do Sul & 8 & $6,6 \%$ \\
Rio de Janeiro & 7 & $5,8 \%$ \\
Santa Catarina & 4 & $3,3 \%$ \\
Espirito Santo & 3 & $2,5 \%$ \\
Bahia & 2 & $1,7 \%$ \\
Ceará & 1 & $0,8 \%$ \\
Goiás & 1 & $0,8 \%$ \\
Paraná & 1 & $0,8 \%$ \\
Rio Grande do Norte & 1 & $0,8 \%$ \\
Rondônia & 1 & $0,8 \%$ \\
Chile* & 1 & $0,8 \%$
\end{tabular}

* de forma desconhecida um participante chileno respondeu à pesquisa.

A grande maioria $(73,5 \%)$ dos cantores que responderam ao estudo foi de homens, com apenas 32 mulheres (26,5\%); A média de idade ficou entre 16 e 58 anos (média 32,9). 50 cantores eram da cidade de São Paulo (41,3\%) e o maior número de respostas se deu nas regiões Sul e Sudeste do Brasil (94,2\%).

A tabela 2 mostra os gêneros musicais que os respondentes cantam. Foram 341 respostas no total, ja que foi solicitado aos participantes que dissessem até três gêneros que costumam cantar. Somando todos os subgêneros do rock como: hard rock, classic rock, grunge, entre outros, sem incluir o metal, o gênero rock foi mencionado 103 vezes $(30,2 \%)$, sendo este o mais apontado na pesquisa. O segundo grupo mais 
citado foi do metal e seus subgêneros, como: death metal, thrash metal, power metal, etc., (20,2\%). Em terceiro lugar a Música Popular Brasileira - MPB (10,3\%). Apenas 20 (16,5\%) dos 121 cantores não cantam rock e/ou metal ou algum de seus subgêneros, como o hard rock e o death metal.

Citados duas vezes, aparecem os gêneros: belting (conforme apontado pelos participantes), black metal, gothic metal, hardcore, metal melodico, metalcore, power metal, punk rock e stoner rock. Além desses, ainda foram mencionados uma única vez os gêneros: acústico (como descrito pelo participante), backing vocal, canto coral, dark rock, folk rock, funk, gótico, indie, música antiga, música espírita kardecista, postgrunge, post-hardcore, metal progressivo, rap, reggae e rock melódico.

Tabela 2 - Relação dos gêneros musicais citados pelos participantes da pesquisa

\begin{tabular}{|c|c|c|c|}
\hline Gênero musical & $\begin{array}{l}\text { Número de } \\
\text { vezes que o } \\
\text { gênero foi citado }\end{array}$ & $\begin{array}{l}\text { \%* de respostas } \\
\text { entre participantes } \\
\qquad(n=121)\end{array}$ & $\begin{array}{c}\% \text { entre as } \\
\text { respostas } \\
(\mathrm{n}=341)\end{array}$ \\
\hline rock & 57 & 47,1 & 16,7 \\
\hline pop & 45 & 37,2 & 13,2 \\
\hline $\mathrm{mpb}$ & 35 & 28,9 & 10,3 \\
\hline heavy metal & 26 & 21,5 & 7,6 \\
\hline hard rock & 22 & 18,2 & 6,5 \\
\hline metal & 17 & 14,0 & 5,0 \\
\hline blues & 12 & 9,9 & 3,5 \\
\hline gospel & 12 & 9,9 & 3,5 \\
\hline classic rock & 10 & 8,3 & 2,9 \\
\hline erudito & 9 & 7,4 & 2,6 \\
\hline soul & 9 & 7,4 & 2,6 \\
\hline lirico & 8 & 6,6 & 2,3 \\
\hline death metal & 7 & 5,8 & 2,1 \\
\hline jazz & 5 & 4,1 & 1,5 \\
\hline country & 4 & 3,3 & 1,2 \\
\hline folclórica & 4 & 3,3 & 1,2 \\
\hline
\end{tabular}




\begin{tabular}{llll}
\hline musical & 4 & 3,3 & 1,2 \\
popular & 4 & 3,3 & 1,2 \\
rEb & 4 & 3,3 & 1,2 \\
thrash metal & 4 & 3,3 & 1,2 \\
grunge & 3 & 2,5 & 0,9 \\
new metal & 3 & 2,5 & 0,9 \\
\hline
\end{tabular}

Nesta tabela não foram listados os gêneros que apareceram menos de três vezes na pesquisa, esses estão listados no corpo do texto. *O total ultrapassa $100 \%$ por que cada cantor pôde citar mais de um gênero.

Ao nomearem as distorções vocais intencionais (DVIs) que estão habituados a cantar os cantores apresentaram 412 respostas, com 115 termos diferentes. Entretanto, alguns desses (27) são termos usados normalmente para vozes não destorcidas, como: voz de peito, voz mista, voz de cabeça, quebra de voz, falsete (mencionado 11 vezes), vibrato (seis vezes), belting e melisma (três menções cada), open throat technique, etc., e foram considerados como respostas que não se enquadraram no objetivo do estudo.

Outros nove termos não podem ser claramente relacionados a distorções vocais e também foram descartados: apertado, mordido, punch, voz caricata, esganiçada, espremida, voz com constrição ou constringido e termos referentes a soprosidade (citados quatro vezes) e alteração laríngea. Dessa maneira foram excluídos 36 termos e a coleta foi finalizadas com um total de 79 termos claramente relacionados a distorções vocais intencionais para serem analisados.

A tabela 3 mostra que dos 121 cantores, 81 (66,9\%) utilizaram alguma variação com a palavra drive para denominar esse tipo de som cantado. As variações de growl (27,3\%) foram o segundo grupo de termos mais apontado, seguido pelas variações de scream $(23,1 \%)$ e voz rasgada e power creaky (19\% cada).

A palavra creaky é comumente traduzida como voz crepitante, e essas foram consideradas como um termo só, junto com os prováveis erros de grafia: creek, creak, e crack. Mesmo caso de scream e voz gritada. 
Além dos termos indicados na tabela 3, por duas vezes as seguintes expressões foram citadas: caveman growl, drive glótico, drive misto, fry scream/screaming, inhale/som respirado pra dentro, low creaky, pig squeal, power creaky growl, power creaky phaser, rasp, scream phaser, screamo e voz suja.

Quarenta e oito outras expressões foram citadas uma única vez, Foram essas: arranhado, caveman, drive exprexe isguim exprou, drive growl, drive impostado, drive subglótico, drive aberto agudo, drive espremido grave, epiglote, epiglote com false chords, false chords lateral, false chords screaming, garganta, gárgula, gralha, grit, growl com voz ativa, growl com voz passiva, growl estilo blues, growl estilo Louis Armstrong, growl scream, gutural melódico, harsh, high creaky, howl, imitação tosca do James Hetfield, inward, kargyraa, mid creaky, phaser do Rod Stewart, phaser howl, power drive, power fry, power growl, power scream, scream creaky, scream growl, scream screech, snarl phaser, som feito com a mucosa da garganta, tell, tunnel throat, uvular, rasgado do Axl, rasgado do Nazareth e médio sujo

Tabela 3 - Termos citados por cantores para denominar as distorções vocais intencionais.

\begin{tabular}{lcc}
\hline Nome indicado & $\begin{array}{l}\text { Número de vezes em que } \\
\text { o termo foi citado }\end{array}$ & $\begin{array}{l}\%^{*} \text { de cantores que } \\
\text { utilizaram o termo } \\
\text { (n=121) }\end{array}$ \\
\hline Drive & 67 & $55,4 \%$ \\
growl/growling & 27 & $22,3 \%$ \\
Gutural & 26 & $21,5 \%$ \\
creaky/creek/creak/crack/crepitante & 22 & $18,2 \%$ \\
Rasgado & 21 & $17,4 \%$ \\
scream/gritado & 21 & $17,4 \%$ \\
power creaky & 19 & $15,7 \%$ \\
Fry & 18 & $14,9 \%$ \\
Phaser & 16 & $13,2 \%$ \\
distorção/distortion & 5 & $4,1 \%$ \\
Grunt & 5 & $4,1 \%$ \\
false chords & 4 & $3,3 \%$ \\
power phaser & 4 & $3,3 \%$ \\
drive supraglótico & 3 & $2,5 \%$ \\
phaser creaky & 3 & $2,5 \%$ \\
Rattle & 3 & $2,5 \%$ \\
rouquidão/rouco & 3 & $2,5 \%$ \\
\hline
\end{tabular}


Nesta tabela não foram listados os termos que apareceram menos de três vezes na pesquisa, esses estão listados no corpo do texto.

*O total ultrapassa $100 \%$ por que cada cantor pôde citar mais de um termo.

\section{Discussão}

São Paulo é a cidade mais povoada do Brasil, e isso certamente influencia na quantidade de respostas oriundas desta capital no questionário (Tabela 1). Além disso, não é possível negar que boa parte desse resultado se deve ao fato de o autor da pesquisa residir nessa cidade e conhecer uma grande quantidade de cantores na mesma, o que facilitou o contato e aumentou a quantidade de respostas.

O fato de $83,5 \%$ dos participantes serem cantores de rock e ou metal (Tabela 2) também pode ter sido influenciado pelo autor ser cantor de rock e ensinar técnicas do gênero, mas o elevado número indica uma forte relação entre as distorções vocais e esses tipos de música. De fato, efeitos de distorção vocal são normalmente relacionados aos subgêneros do rock (GUZMAN ET AL, 2014; IZDEBSKI ET AL, 2017), mesmo que se saiba que estão presentes no canto há incontáveis anos (LEVIN E EDGERTON, 1999) e que apareçem em todo tipo de música (TITZE, 1998; GRAWUNDER, 2010; MCGLASHAN ET AL, 2013). Essa diversidade de gêneros também ficou demonstrada nas respostas dos cantores que mencionaram música sertaneja, MPB, música folclórica, country, teatro musical, samba, pop e até erudito (Tabela 2).

Como a pergunta foi direcionada ao estilo que cantam (Tabela 2), e não ao estilo que utilizam as DVIs, não foi possível afirmar que todas as respostas que mencionaram erudito, lírico, canto coral, música antiga e MPB têm relação direta com os ajustes distorcidos, tampouco gêneros como o metal melódico de bandas como Helloween e Stratovarius, que por muitas vezes utiliza vozes mais "limpas". De qualquer forma, é interessante verificar que os cantores afirmaram possuir a habilidade de transitar entre gêneros e diferentes produções vocais com e sem o uso das DVIs. 
Sobre os termos utilizados (Tabela 3) ficou claro que a palavra drive é, no Brasil, a mais utilizada para denominar esses tipos de vozes. No país é comum ouvir este termo sendo aplicado até mesmo em reality shows com competição de cantores como Ídolos e The Voice. Não se sabe a origem exata e nem é possível afirmar por qual razão drive é tão popular no Brasil. O termo, em inglês, significa unidade, dirigir, mas é possível que tenha surgido a partir dos amplificadores e pedais de efeito de guitarra elétrica, nos quais a função overdrive, quando acionada, gera distorção no som do instrumento (YEH ET AL. 2007), e tal efeito é comum em gêneros como rock e metal.

Fator que vale mencionar, é que não se encontra esse termo em publicações internacionais, tampouco em conversas com profissionais extrangeiros eles reconhecem tal termo, mesmo sendo uma palavra em inglês. Nem mesmo o cantor chileno que preencheu o questionário utilizou drive. Diferente de growl, que aparece em publicações e métodos de canto em diversos países (SAKAKIBARA, 2007; MCGLASHAN ET AL. 2013; GUZMAN ET AL., 2014) e pode ser traduzido como rosnado, o que remete à sonoridade animalesca que esse tipo de canto pode trazer.

Os outros termos mais utilzados foram scream, voz rasgada e power creaky (Tabela 3). Scream significa grito e é um termo comum no Brasil e fora, e, apesar de distorções vocais serem técnicas realizadas conscientemente após treinamento direcionado, muitas vezes o objetivo dos cantores é soar como se estivessem gritando, mesmo que não estejam de fato.

Voz rasgada remete a sensações auditivas, pois DVIs podem ter características perceptivas como de um papel ou tecido sendo rasgado. Já power creaky é um termo que foi criado por um método de canto específico para ensinar distorções vocais, e tem como explicação o fato de soar como uma voz crepitante, porém mais forte e poderosa, daí o nome, que pode ser traduzido como crepitação poderosa.

No total, 121 cantores responderam o questionário, e este estudo considerou dentro do objetivo 78 termos diferentes para as DVIs (Tabela 3). Entretanto, dificilmente pode-se afirmar que existam 78 possibilidades de ajustes no 
trato vocal capazes de merecerem um nome cada. Em algumas respostas dadas é possível argumentar que existem variações mínimas que produzem sonoridades distintas como nos casos do participante que respondeu growl com voz ativa e growl com voz passiva, ou o que mencionou gutural e gutural melódico. A impressão que esses dois casos passam é que existe um ajuste com produção de uma nota músical perceptível (frequência fundamental) em um ajuste e no outro não, o que pode dar a sensação "som melódico", ou que um dos ajustes seja realizado com maior contato das pregas vocais (HERBST ET AL., 2015) que o outro, dando a sensação de "voz ativa". Casos como as variações de creaky, phaser, power creaky, scream, growl, citados pelos mesmos cantores também evidenciaram essa proximidade de ajustes com variação mínima.

Alguns métodos comerciais de canto (SADOLIN, 2012; COELHO, 2017) têm como objetivo justamente o controle dessas pequenas variações dentro de uma mesma forma de produção de distorção vocal. Nota-se, pelos termos utilizados nas respostas, que muitos dos estudantes desses métodos participaram desta pesquisa. Tais métodos acabam por utilizar expressões que já são comuns no meio ou optam por criar algumas outras por distintas razões. Essas razões podem ser: fisiológicas, quando esses estão aliados a pesquisas científicas; pedagógicas, quando buscam formas para facilitar a comunicação com os alunos; ou mesmo por razões de mercado, quando querem passar a impressão de se diferenciarem de outros produtos já disponíveis.

Expressões relatadas (Tabela 3) como power creaky e phaser em sua maioria se deveram a uma metodologia brasileira de canto rock (COELHO, 2017), e outras como rattle e distortion ganharam mais fama após serem utilizadas em um método dinamarquês de canto (SADOLIN, 2012) bastante famoso na Europa e estados Unidos. Grit e scream são comuns, e foram utilizadas por Vendera (2012) e fry scream e false chords também estão presentes nos trabalhos de uma professora americana (CROSS, 2007). Não é possível afirmar a origem de nenhum desses termos, exceto o fry scream, que a autora Cross (2007) afirma ter inventado.

O fato de haver métodos tentando sistematizar a prática do canto distorcido é sinal de que cantores estão buscando entender e se aprimorar nesses 
assuntos, mas em alguns casos ainda encontramos aqueles que não conseguem nomear o som que produzem, e acabam utilizando frases como: o "som feito na mucosa da garganta", que, apesar de utilizar uma linguagem anatômica, não traz nenhuma informação de fato.

Em alguns casos, na hora de denominar o som, cantores remetem a imitações de famosos, com: "imitação tosca do James Hetfield" (cantor da banda Metallica) ou o "rasgado do Axl" (Axl Rose, vocalista da banda Guns n' Roses) e o "rasgado do Nazareth" (Nazareth, banda de rock dos anos 1970). Ambos são sons "rasgados", porém com alguma variação sentida pelo cantor na hora de produzir e ouvir a emissão. Surpreendentemente, essas formas de mencionar as distorções foram citadas poucas vezes. No meio dos cantores é comum relacionar o tipo de voz a de um cantor famoso que sirva como referência, especialmente quando se questiona, por exemplo: "como faz a voz do David Coverdale?" (cantor da banda Whitesnake), ou "como é o drive do Chris Cornell?" (vocalista falecido que ficou famoso nas bandas Soundgarden e Audioslave).

Growl, scream e gutural são formas de nomear efeitos distorcidos sem uma origem certa. Growl e scream são comuns em cursos de canto que ensinam esses tipos de ajustes (SADOLIN, 2012; COELHO, 2017) e gutural é bastante usual mesmo para quem não canta, principalmente entre fãs de heavy metal e subgêneros.

Existem ainda termos que não parecem ter sentido fisiológico, como o "drive subglótico", já que não há nada abaixo da glote que possa produzir tais efeitos. Outros têm grande influência das percepções táteis e visuais, como na voz rasgada, voz suja e voz arranhada.

Alguns termos que não têm relação com distorções vocais apareceram nas respostas de alguns dos participantes, como: melisma, falsete, voz de cabeça, whistle, yodel, pianíssimo, lírico, etc. Das 66 vezes em que foram mencionados esses tipos de termos, 34,8\% foram citadas por cantores que não cantavam rock. Se considerarmos o pop rock como subgênero do pop e não do rock, esse número sobe para 51,5\%. Ou seja, mais da metade dos termos referidos a vozes não distorcidas foram indicados pelos 16,5\% de participantes que não estão acostumados aos gêneros musicais que mais apareceram na pesquisa, o rock e o metal. Talvez esse dado seja mais um indicativo 
da representatividade das distorções vocais nesses gêneros. Claro que é possível que se essa pesquisa fosse realizada por cantores na região de Tuva e da Mongólia, famosas pelo canto tradicional kargyraa, que é distorcido (LINDESTAD ET AL., 2001), o resultado fosse outro.

Esse fato, somado à grande quantidade de termos apresentados mostra o quanto ainda é necessária a investigação e divulgação de material especializado nesse tipo de ajuste, para que cantores possam se comunicar e se entender ao falarem de vozes distorcidas. Talvez seja confusão semelhante a existente sobre o uso ou não de falsete, sobre o que é belting, quem faz ou não faz twang, o que é respiração diafragmática e outros embates comuns no mundo do canto.

Atualmente existem inúmeras fontes de informação a respeito das distorções vocais, sejam cantores que repassam suas experiências pessoais, professores que criam métodos comerciais ensinando DVIs ou pesquisadores que investigam a voz cantada, mas nem sempre essas fontes estão em sintonia. Essa falta de consenso em como vozes distorcidas devem ser definidas ou nomeadas dificulta a comunicação e compreensão do que está estudado ou discutido e apareceu de forma clara nesta investigação.

Em resumo, a presente pesquisa investigou cantores que utilizam vocais distorcidos em suas músicas e encontrou 78 termos diferentes entre os 121 participantes, sendo os mais utilizados: drive, growl, gutural, variações de creaky, rasgado e scream.

O fato de terem sido mencionados tantos termos para as distorções vocais indica o quão importante é que sejam realizados mais avanços nos estudos desses tipos de vozes, além de uma melhor comunicação e integração entre os profissionais que atuam no meio.

Uma pesquisa fora do Brasil com cantores, professores de canto e demais profissionais da voz será realizada para verificar se há similaridade entre os termos adotados. 


\section{Referências Bibliográficas}

BORCH, D. Zangger et al. Vocal fold vibration and voice source aperiodicity in 'dist' tones: a study of a timbral ornament in rock singing. Logopedics Phoniatrics Vocology, v. 29, n. 4, p. 147-153, 2004.

COELHO, Ariel. Drives Vocais - Memórias de Acesso. Hotmart. 2017. Disponível em: <https:/ / drivesvocaismemoriasdeacesso.club.hotmart.com/lesson/qV7yEwAo7J/4os-drives-vocais-e-as-contribuicoes-da-antropofisiologia-vocal>. Acesso em: 26 fev. 2018.

CROSS, Melissa. Zen of Screaming. Direção: Denise Korycki. New York: Loudmouth Inc, 2007. 1 DVD e 1 CD (145 min). NTSC, color.

GRAWUNDER, Sven. On the Physiology of Voice Production in South-Siberian Throat singing-Extended Abstract. Phonetician. V. I/II. n. 101/102. p. 25-32. 2010.

GUZMAN, Marco et al. Laryngoscopic, acoustic, perceptual, and functional assessment of voice in rock singers. Folia Phoniatrica et Logopaedica, v. 65, n. 5, p. 248256, 2014.

HERBST, Christian T. et al. Glottal adduction and subglottal pressure in singing. Journal of Voice, v. 29, n. 4, p. 391-402, 2015.

IZDEBSKI, Krzysztof. 3D video reconstructions of vocal folds in extreme heavy metal (growl) singing from HSDP. ePhonoscope, v. 1, n. 1, 2017.

LEVIN, Theodore C.; EDGERTON, Michael E. The throat singers of Tuva. Scientific American, v. 281, n. 3, p. 80-87, 1999.

LINDESTAD, Per-Åke et al.. Voice source characteristics in Mongolian "throat singing" studied with high-speed imaging technique, acoustic spectra, and inverse filtering. Journal of Voice, 15(1), 78-85. 2001.

MCGLASHAN, Julian; SAYLES, Mark; SADOLIN, Cathrine. Vocal effects in singing: a study of intentional distortion using laryngostroboscopy and electrolaryngography. In 10th International Conference Advances in Quantitative Laryngology. Cincinnati, EUA. p. 81, jun. 2013.

NEUBAUER, Jürgen; EDGERTON, Michael; HERZEL, Hanspeter. Nonlinear phenomena in contemporary vocal music. Journal of Voice, v. 18, n. 1, p. 1-12, 2004.

POMPEU, Ana Terra Santos; BARREIRO, Suamit Marques. Rua, que a nossa voz seja ouvida: uma contribuição fonoaudiológica sobre a voz no Rap nacional. Música Popular em Revista, v. 2, 2017. 
SADOLIN Cathrine. Complete Vocal Technique. Dinamarca: Cvi Publications, 2012.

SAKAKIBARA, Ken Ichi et al. Observation of subharmonic voices. Proceedings of the ICA, Madrid, Spain. 2007.

TITZE, Ingo. Deliberate use of distortion in singing. The Journal of the Acoustical Society of America, v. 103, n. 5, p. 2796-2797, 1998.

VENDERA, Jaime. Raise Your Voice: the advanced manual. Ohio, EUA: Vendera Publishing, 2013.

YEH, David T.; ABEL, Jonathan S.; SMITH, Julius O. Simplified, physically-informed models of distortion and overdrive guitar effects pedals. In: Proc. of the Int. Conf. on Digital Audio Effects (DAFx-07). Bordeaux, França. p. 10-14. 2007.

YOSHINAGA, Ikuyo; KONG, Jiangping. Laryngeal vibratory behavior in traditional Noh singing. Tsinghua Science and Technology, Pequim, China. v. 17, n. 1, p. 94-103, 2012. 